\title{
Targeting Existing Power Plants: EPA Emission Reduction with Wind and Demand Response
}

\author{
J. B. Cardell ${ }^{\mathrm{a}, *}$, C. L. Anderson ${ }^{\mathrm{b}}$ \\ ${ }^{a}$ Smith College, Northampton, MA \\ ${ }^{b}$ Cornell University, Ithaca, $N Y$
}

\begin{abstract}
Electricity generation accounts for $40 \%$ of $\mathrm{CO}_{2}$ emissions from fossil fuel combustion in the United States. Section 111 of the Clean Air Act (CAA) allows for greenhouse gas emission regulation by the US Environmental Protection Agency (EPA). In June, 2014, EPA issued the Clean Power Plan that proposes regulation of existing power plants via a "best system of emission reduction" or BSER. Reducing carbon dioxide emissions caused by electricity generation is one of the main motivations for increasing wind power and other renewable energy use, and this option is included in the BSER. This paper applies Monte Carlo simulation with a two-stage power flow optimization framework to analyze the potential $\mathrm{CO}_{2}$ emission reduction with $10 \%$ and $20 \%$ wind penetration using the proposed BSER. The results show that EPA's BSER does achieve significant emission reduction, but an increase in cost of electricity and load curtailment can result if significant wind is installed without other measures. These concerns are eliminated by including recourse to real-time demand response along with EPA's BSER, suggesting that the proposed BSER, implemented alone, could be insufficient for reaching EPA's target $\mathrm{CO}_{2}$ reductions while also safeguarding power system reliability and cost.
\end{abstract}

Keywords: $\mathrm{CO}_{2}$ emission reduction, BSER, wind integration, demand response, carbon price, Monte Carlo simulation

\section{Introduction}

\subsection{Policy Background}

Reducing carbon dioxide emissions caused by electricity generation is a driving motivation for increased wind power and other renewable energy installations. In the United States, state governments have promoted the growth of renewable energy through renewable portfolio standards, RPS, (DSIRE (2014); Barbose (2012); Heeter et al. (2014)), and in some cases, carbon emissions limits

\footnotetext{
* Corresponding author

Email address: jcardell@smith.edu (J. B. Cardell)
}

Preprint submitted to Energy Policy

January 15, 2015 
(RGGI (2012); AB32 (2006); CACJA (2010)). The federal government has also supported the use of renewables through the production tax credit and other incentives (UCS (2014); DSIRE Solar Incentives (2014)). In June 2014, a significant advance was achieved in limiting greenhouse gas emissions when the US Environmental Protection Agency, EPA, published a proposed rule-making for regulating carbon emissions from existing power generators, under $\S 111(\mathrm{~d})$ of the federal Clean Air Act. Along with a rulemaking in January 2014 for regulating carbon emissions from new generating units, these two policies represent the first time that the United States federal government has acted to limit $\mathrm{CO}_{2}$ emissions. To achieve the target reductions, the Clean Power Plan (US EPA (2014a) ) for existing generating units defines a "Best System of Emission Reduction," BSER, composed of four building blocks, one of which is increased use of zero-carbon emitting sources.

The research presented here analyzes the role of wind power in reducing power system carbon emissions with other building blocks in EPA's BSER plus real-time demand response. This paper also aims to be part of broader industry discussions on the benefits and drawbacks of widespread integration of renewables and demand-side programs. The intent is that the work presented here, both the modeling framework and the results, will inform industry stakeholders and researchers involved with reducing pollutant emissions for the electric power sector. As highlighted in the results section, the elements of EPA's proposed BSER achieve the best emission reduction when implemented together, as a system. These results are anticipated to be useful to policy makers, regulators, system operators, renewables developers and load-aggregators, as these groups work together in further specifying, promoting and implementing the BSER.

The Clean Power Plan evolved from a 1999 petition filed with EPA for federal regulation of six specific GHGs (NACAA (2011)). Responding to this petition in 2003, the EPA found that it did not have the authority to regulate GHGs, and therefore GHGs were not air pollutants, under the CAA. A subsequent effort toward federal GHG regulation came in 2005 when Massachusetts with other states, cities and organizations sued EPA to force regulation of greenhouse gases (US Supreme Court (2005)). In 2007 the US Supreme Court ruled in favor of Massachusetts stating that GHGs are to be considered air pollutants and are subject to regulation under the CAA. Subsequently applying the finding (US Supreme Court (2005)) to stationary sources, EPA, in 2014 for the first time, began to regulate GHG emissions from electric power plants under $\S 111$ of the Clean Air Act.

Section 111 provides for distinct methods of $\mathrm{CO}_{2}$ regulation to be developed for new generating units. Section 111(b) directs the EPA to establish federal standards for $\mathrm{CO}_{2}$ emissions from new units that define the level of emission limitation achievable, taking into account costs and health impacts (US EPA $(2014 \mathrm{e})$ ). For new sources, separate $\mathrm{CO}_{2}$ emissions standards are defined for each fossil fuel generating technology, and each new generating unit must meet these technology-specific federal standards.

Regulation of existing generating units is significantly more difficult. In contrast to the rules for new sources, a proposed rulemaking for existing sources 
published in June 2014, the Clean Power Plan (US EPA (2014a)), does not prescribe specific emission limits. EPA recognized that existing plants may not be able to be retrofitted to meet technology-specific emissions standards at reasonable cost. Therefore, the proposed rulemaking for existing generating units provides for greater flexibility in meeting $\mathrm{CO}_{2}$ emissions reductions. EPA has the authority to create guidelines at the federal level that individual states then apply in setting the $\mathrm{CO}_{2}$ emissions standards for existing fossil fuel-fired generating units. EPA's guidelines define a best system for emission reduction, as discussed in Section 1.2.

\section{2. $\mathrm{CO}_{2}$ Emission Reductions From Existing Sources}

The Clean Power Plan (US EPA (2014a)) sets a nation-wide target of 30\% reduction in $\mathrm{CO}_{2}$ emissions from the power sector by 2030, from the 2005 level. EPA's emission reduction guidelines for existing generators are in what EPA has termed the "Best System of Emission Reduction," or BSER. Focusing on $\mathrm{CO}_{2}$ emissions, and intending that other GHGs will be reduced along with $\mathrm{CO}_{2}$, the BSER consists of four building blocks:

1. Decrease fossil fuel-fired generating unit carbon intensity by decreasing the unit's heat rate,

2. Reduce system-wide emissions, through system re-dispatch to lower carbon intensive units (e.g., replace coal generation with that from natural gas combined cycle plants),

3. Reduce system-wide emissions through re-dispatch to zero-carbon emitting plants (e.g., renewable energy or nuclear sources),

4. Reduce system-wide emissions through expanded use of demand-side energy efficiency programs.

In defining the BSER, EPA envisions emission reductions to be achieved regionally rather than unit-by-unit. Though nominally focused at the state level, the requirements of the Clean Power Plan can be met through multi-state programs. This flexibility provided for meeting the federal $\mathrm{CO}_{2}$ emission guidelines has also been criticized: in allowing for reductions to be facilitated by measures "outside the fence" (of the power plant), the federal guidelines can expect greater $\mathrm{CO}_{2}$ emission reductions than if measures were restricted to those possible "inside the fence," (Eilperin and Mufson (2014)). Opponents of GHG regulation seek to minimize emission reduction expectations.

In defining the nation-wide reduction target, EPA developed a range of expected state-specific targets (US EPA $(2014 \mathrm{a}|\mathrm{b}| \mathrm{d} \mid \mathrm{f})$ ). The test power system used for the analysis in this paper nominally represents the New England region, so these states are referenced in the discussion below. However, these results are relevant to illustrate the extent of variation expected in meeting the EPA nation-wide emission goal.

Table 1 shows the 2030 final goals for reductions in $\mathrm{CO}_{2}$ for the New England states and the states with the minimum and maximum goals, North Dakota and Washington state (US EPA $(2014 \mathrm{~d}))$. This table shows the percent reduction 
and the absolute lbs- $\mathrm{CO}_{2} / \mathrm{MWh}$ that will be achieved by these reductions. Column 2 indicates the adjusted output-weighted-average $1 \mathrm{bs}-\mathrm{CO}_{2} /$ net-MWh from all affected fossil fuel-fired generating units. EPA explains that if a state installs renewable energy plants or expands demand-side energy efficiency, and in turn decreases the number of hours a coal plant is dispatched, the decreased emissions from the reduced coal-fired generation represents the "adjusted output" in the target lbs- $\mathrm{CO}_{2} / \mathrm{MWh}$.

Tables 2 and 3 contain further details of the BSER. Table 2 (US EPA (2014a) table 6) shows the target generation from renewable energy in the states. Comparing Maine and New Hampshire, this table shows that Maine already has significant generation expected from renewable sources, and with growth in electrical demand through 2030, the percent of generation from renewable resources actually decreases. This accounts for both the low target decrease in emissions and the low lbs- $\mathrm{CO}_{2} / \mathrm{MWh}$ in Table 1. New Hampshire is expected to have significant growth in renewable energy generation, consistent with the high target $\mathrm{CO}_{2}$ reduction from Table 1 . This discussion illustrates the regional flexibility envisioned for achieving the nation-wide reduction of $30 \%$ in $\mathrm{CO}_{2}$ emissions.

Turning to demand-side energy efficiency, Table 3 (US EPA (2014a) table 7, US EPA (2014c) table 5-4) shows these targets, revealing relatively consistent expectations by EPA across the states. Details of the EPA state goal calculations are in (US EPA $(2014 \mathrm{~d}$ b f $))$.

This paper analyzes the potential for power system-wide $\mathrm{CO}_{2}$ emission reductions from existing generating units, consistent with the EPA proposed Clean Power Plan rulemaking under CAA $\S 111(\mathrm{~d})$, focusing in particular, but not exclusively, on the role of wind power in facilitating these reductions. Analysis of wind power is used here to address issues of intermittency and uncertainty, without suggesting a lesser importance for the potential of solar power. Section 2 describes the methods for modeling the BSER building blocks in this paper. Section 3 introduces the two-stage optimization framework developed for analyzing system $\mathrm{CO}_{2}$ emissions, using the IEEE 39-bus test power system and defines the modeling scenarios. Section 4 discusses the results from simulating the test system with wind power, energy efficiency, demand response, and a carbon price, with discussion and policy implications provided in Section 5.

\section{Methods: Representing BSER Building Blocks}

For new generating units, EPA expects the best available technologies to be installed as part of controlling $\mathrm{CO}_{2}$ emissions. For existing generators, EPA states "that the most cost-effective system of emission reduction for GHG emissions from the power sector ...entails not only improving the efficiency of fossil fuel-fired [generators], but also addressing their utilization [through] opportunities for lower-emitting generation and reduced electricity demand" (US EPA (2014a), pp 14-15). The measures intended to reduce generator utilization are proposed in the BSER as building blocks 2, 3 and 4 .

The modeling in this paper represents the hundreds of generators in New England as aggregated units of each technology, located at one of the 11 gener- 
ation buses in the test system (see Figure 1) (ISOne (2013); Zimmerman et al. (2010); Bills (1970)). Since actual generating units are not represented in this analysis, building block 1 for improved heat rates at individual units is not modeled. Instead, the analysis focuses on examining the efficacy of the remaining elements of EPA's BSER, the building blocks that focus on decreasing the utilization of fossil fuel-fired units.

\subsection{Building Block 2: Redispatch via a Carbon Price}

The Clean Power Plan building block 2 focuses on "Reducing emissions from ... carbon-intensive ... EGUs [electric generating units] ... [with] generation ... from less carbon-intensive ... EGUs." (US EPA (2014a) p33). The goal of this option is to modify the system dispatch so that lower carbon-intensive units are used more often and higher carbon-intensive units are used less. This change in dispatch order can be achieved by shifting the relative marginal costs of the generating technologies. One method to achieve this shift would be through a cap-and-trade market, employing market forces to impose an implicit cost on burning fossil-fuels. This same shift in dispatch order can be achieved through direct application of a carbon price, represented as $\$ /$ ton $-\mathrm{CO}_{2}$. A carbon price is employed in this paper, with no intention of promoting a carbon price over a cap-and-trade market, but rather to facilitate analyzing the effect on the dispatch order and emission reduction of a carbon price, whether this price is determined by market forces or via a regulatory process.

Analyzing the amount of carbon released through electricity generation begins with analyzing the available technology mix. Using data from (RDI/FT Energy Inc. (2006)), taken from FERC 714 filings (FERC (2000-2014)), Table 4 shows the proportion of coal-fired, natural gas-fired (NGCC and CT units), oil-fired, nuclear and hydro-electric generation modeled in the test system, with 'North' including Maine, New Hampshire and Vermont, 'Mass' showing the capacity in Massachusetts, and 'South' including Connecticut and Rhode Island. The total generating capacity in the test system is scaled to approximately $14 \%$ of the generating capacity in the New England region. This table shows that oil-fired generation has a significant presence, with capacity less than that of natural gas but more than coal-fired generation (ISOne (2013, 2007)). The New England technology mix is known historically to include more oil-fired generation than other regions. Similarly across the country, various areas are known to have proportionally more coal, hydro-electric, and more recently wind power, than other regions. The analysis and results presented in this paper are relatively unaffected by the presence of oil-fired generation since this technology does not affect the relative costs of coal and natural gas, with or without a carbon price. In addition, the contribution of oil-fired capacity versus the actual generated energy, on average, is between $1 \%$ and $2 \%$, both in these simulations and in the actual region (ISOne (2013, 2007)).

The carbon content in fuel is shown in Table 5 , along with fixed and variable generator costs (US DOE/EIA (2012)). The bottom row shows variable costs adjusted for a forecasted potential carbon price of $\$ 52 /$ ton (levelized across the 
study period of Luckow et al. (2014)), a price that would achieve the shift in dispatch order between coal and natural gas units, as discussed above.

Calculation of the $\mathrm{CO}_{2}$ emissions from the generating units in this analysis follows the method and information from (US EPA and OAR (2011)). In particular, the calculation follows

$$
E_{\mathrm{CO}_{2}}=F C \times C C \times F O \times \beta
$$

where $E_{\mathrm{CO}_{2}}$ is $\mathrm{kg}$ of $\mathrm{CO}_{2}$ emissions, $\mathrm{FC}$ is the fuel combusted (US EPA and OAR (2011)), CC is the carbon content coefficient of the fuel, FO is the fraction of carbon oxidized and $\beta$ is the ratio of the molecular weight of carbon dioxide to carbon (US EPA and OAR (2011)). FC is determined from the averaged heat rate for the aggregated generating units and the output of that unit as determined by the system simulations. This analysis assumes that $100 \%$ of the carbon is oxidized, and $\beta=44 / 124$ (US EPA and OAR (2011)). The product of the average regional heat rate, the carbon content coefficient and the molecular conversion to $\mathrm{CO}_{2}$ is shown in the top row of Table 5 .

The study performed in (Luckow et al. (2014) ) develops various forecasts for a future carbon price. The one selected for use in this study is the one that induces a change in the merit order of NGCC units in favor of coal units. As fuel prices also are volatile, the costs reported in Table 5 are not intended to represent future fuel costs. The modeling framework utilized here is developed to have a base case system in which relatively high-polluting, low-cost generation is dispatched before other technologies. This allows for analysis of $\mathrm{CO}_{2}$ emission reduction as the strategy in building block 2 is pursued.

\subsection{Building Block 3: Utilization of Wind Power}

The third building block in EPA's BSER is: "Reducing emissions from affected EGUs in the amount that results from substituting generation at those EGUs with expanded low- or zero-carbon generation" (US EPA (2014a) p34). This option is modeled by adding wind power to the test system shown in Figure 1. As of 2013 , approximately $40 \%$ of the new capacity in the queue for New England was for wind power projects, totalling 1,950MW on-shore and 503MW off-shore (Black (2013)). For this project, four wind farms are modeled, three on-shore and one off-shore using data from (NREL (2010)). Both 10\% and $20 \%$ wind penetration are modeled, with the capacities shown in Table 6 . The exact capacities for each site are determined by aggregating EWITS wind farms that are geographically close to create the required capacity at each bus. As a result of using the existing wind farm data the total capacities are approximately, but not exactly, $10 \%$ and $20 \%$ of the installed regional capacity. The simulation scenarios using the different levels of wind power are delineated in Section 3 .

\subsection{Building Block 4: Demand-Side Energy Efficiency}

The final element in EPA's BSER is: "Reducing emissions from affected EGUs [through] the use of demand-side energy efficiency" (US EPA (2014a) 
p34). For this element, the modeling framework includes energy efficiency measures through reducing system load proportionally across the network. The amount of efficiency introduced varies across scenarios via the optimization process, reflecting the greater motivation to promote such measures (and also to pay for them) as net system variability increases with wind penetration. For the analysis presented below, the amount of energy efficiency utilized across the system in different scenarios ranges from $3.1 \%$ to $11.5 \%$, consistent with the information in Table 3 . These values represent a percent decrease from the load level of the given scenario, low, medium or high, as defined below in Table 8 For energy efficiency it is assumed that the reduction in demand is consistent across the hours, as would be expected for improved efficiency of motors, air conditioners, lighting, etc.

\subsection{Facilitating BSER: Real-Time Demand Response}

As wind penetration increases, the system may be exposed to increased net load variability. If the the system is unable to respond to this variability with supply-side generation, it must resort to load curtailment. This insufficient supply to meet demand represents a threat to reliability, and therefore simulation results that include the use of interruptible load indicate a decrease in system reliability in the given system simulation.

To alleviate forced load curtailment, the use of real-time demand response, as made available in real-time regional electricity markets (FERC (2014); Klobasa (2010); Perlstein et al. (2012)) is found to be effective in restoring the supplydemand balance and ensuring system reliability. As modeled here, the real-time demand response is idealized as load responding to the price of electricity in real-time whenever that price is greater than or equal to $\$ 100 / \mathrm{MWh}$. This is in contrast to existing demand response programs that typically are restricted in the number of demand response events per year. The demonstration of benefits of the real-time demand response based on real-time pricing, as modeled here, are intended to motivate industry stakeholders to move toward real-time pricing for demand response.

Though the Monte Carlo simulations discussed in Section 3 represent hundreds of thousands of possible realizations of load and available generating capability, the simulations are not sequential. Therefore, though it is possible that the load would be shifted to other hours, that shift is not modeled here, and so emission reduction could be over-represented in our results. However, the main role of the real-time demand response (as opposed to forced load curtailment or energy efficiency) in this modeling effort is its positive impact in improving system reliability, and not its role in emission reduction.

To compare the implementation of load curtailment versus real-time demand response, note that interruptible, or curtailable, load is modeled as available for curtailment at a price of $\$ 10,000 / \mathrm{MWh}$. This price is a proxy for the value of lost load, and is set to provide an economic incentive to use generation to meet system demand, while allowing load curtailment if no generation is available (Mount et al. (2011); Willis and Garrod (1997); Murillo-Sanchez et al. (2013)). If real-time demand response is available, as a less expensive alternative to 
forced load curtailment, the price that triggers response is set to $\$ 100 / \mathrm{MWh}$. These prices allow the system simulation to curtail load as needed, with a clear indication that reliability was threatened whenever the electricity price spikes to $\$ 10,000$. The price of $\$ 100 / \mathrm{MWh}$ for demand response is consistent with historical values for the New England region, and within the range of generation costs, making demand response an attractive yet not low cost option (ISOne (2007, 2006); Ballantine and Cooke (2014)). The total allowed penetration of efficiency plus demand-response is up to $15 \%$ of demand, consistent with analyses by the federal government and the New England system operator (FERC (2014, 2012); NERC (2013)).

\section{Methods: Two-stage Modeling Framework}

In order to analyze the impact of BSER policies on $\mathrm{CO}_{2}$ emissions from the electricity sector, a model must be developed that incorporates the power network, market mechanisms, consumer demand and variable generation with realistic uncertainty. With this model, the proposed policies can be simulated to ascertain likely performance. The simulation of the power system and market mechanisms are achieved through a two-stage stochastic optimization framework, representing the two time periods to incorporate uncertainty in wind and demand forecasts. In the following subsections we describe each of the stages and the linkages between them. The interested reader is referred to Anderson and Cardell (2013) for more detail.

\subsection{Stage 1: Hour-Ahead Dispatch}

The first stage of the model simulates the hour-ahead decisions made by the system operator. At this time, the system operator has knowledge of the available generators, their capacity and costs. In addition, there exists a forecast for the expected wind generation one hour in the future. With this information, the system operator can use the AC optimal power flow (OPF) formulation, given in Equation (2), to determine the generation/dispatch plan for the next hour.

$$
\begin{aligned}
& \min _{x} \sum_{j=1}^{N_{G}} C\left(P_{j}^{G}(x, \xi)\right)+C\left(Q_{j}^{G}(x, \xi)\right) \\
& \text { subject to } x \in \mathcal{C}_{\text {stat }}^{\text {gen }} \cap \mathcal{C}_{\text {stat }}^{\text {tr }} \\
& f(x, \xi)=0
\end{aligned}
$$

where $C(\cdot)$ represents cost functions for real and reactive power, respectively. The vector $x$ represents all parameters for voltage and power at each bus including $V_{m}, \Theta_{v}, P_{G}$ and $Q_{G}$, and the vector $\xi$ represents the forecasted wind output. The set $\mathcal{C}_{\text {stat }}^{\text {gen }}$ represents the static operating constraints for all generators, including minimum and maximum operating limits, $\mathcal{C}_{\text {stat }}^{\mathrm{tr}}$ represents network transmission limits, and $f(x, \xi)$ represents the power balance constraint. 


\subsection{Stage 2: Real-Time Dispatch}

The time period referred to as "real-time" can represent the five- or tenminute market depending on the specific system of interest. This time period is the second stage of the optimization problem, wherein wind generation is realized, and recourse is available through the adjustment of generator dispatch and demand side resources. As a result of the short time available for adjustment of output, the ability to increase or decrease generation is constrained by unit ramp limits. Therefore the ability of the system to adapt to forecast errors in variable generation, or forecasted demand is fairly limited. The generator ramping capabilities are shown in Table 7 (Shahidehpour and Wang (2004); Lalor and O'Malley (2003); NPCC (2002); Shrestha et al. (2004); Hamal and Sharma (2011); Lindsay and Dragoon (2010)).

At this time, the OPF problem is re-solved, with addition of a new set of dynamic constraints on generators $\left(\mathcal{C}_{\text {dyn }}^{\text {gen }}\right)$ to impose the physical ramp limits of generators mentioned above. Note that the parameter $\xi$ is replaced with $\omega$ as in Equation (3),

$$
\omega^{k}=\xi^{k}+\mathbf{P}_{\mathrm{wind}}^{\mathrm{k}}
$$

which is the vector of realized wind generation. With an updated constraint set, the ac OPF model becomes:

$$
\begin{aligned}
& \min _{x} \sum_{j=1}^{N_{G}} C\left(P_{j}^{G}(x, \omega)\right)+C\left(Q_{j}^{G}(x, \omega)\right) \\
& \text { subject to } x \in \mathcal{C}_{\text {stat }}^{\text {gen }} \cap \mathcal{C}_{\text {dyn }}^{\text {gen }} \cap \mathcal{C}_{\text {stat }}^{\text {tr }} \\
& f(x, \omega)=0
\end{aligned}
$$

\subsection{Capturing Uncertainty}

One of the most significant challenges of renewable energy sources is the uncertainty. While wind (and solar) outputs can be forecasted, there is error inherent in the forecasts, which is resolved as the system moves closer to realtime dispatch. Both the forecast error and the variability of wind resources places a burden upon the system operator to use more controllable generation units to maintain the balance between total generation and total demand.

Uncertainty also exists in traditional generation through unexpected 'forced' outages that take some or all of a particular generation unit offline. In this case, the burden of meeting the shortfall is placed with the remaining available generators.

The uncertainty in wind generation is captured through the updating of the forecast vector $(\xi)$, with forecast errors sampled from an empirical distribution based on wind data from (NREL (2010) ) and an autoregressive forecast model. The forecast model, discussed in detail in (Cardell and Anderson (2010)), exhibits relative root mean square error (rel-RMSE) of less than 0.5 , which is within accepted range Lange (2005). Load forecast errors are obtained through 
the implementation of the neural network load forecast model, described in (Tee et al. (2009)).

$$
\begin{aligned}
& P_{D}^{\mathrm{k}} \sim \mathbf{F}_{\text {demand }}(\cdot) \\
& \mathbf{P}_{\text {wind }}^{\mathrm{k}} \sim \mathbf{F}_{\text {wind }}(\cdot) \\
& I_{\mathrm{FOR}}^{j k} \sim \mathbf{F}_{\mathrm{FOR}}^{j}(\cdot)
\end{aligned}
$$

The specific use of the distributions in equations 5 through 7 is illustrated in Figure 2, which shows one simulation of the optimization framework. For each simulation, a sample is drawn from the distributions to represent realistic forecast errors in wind and electricity demand, and potential failures of generating units described above.

In order to examine overall impact of implemented policies over many possible realizations, a Monte Carlo simulation approach is implemented wherein the hour-ahead optimization and real-time adjustment sequence is simulated repeatedly for a large set of samples, covering all combinations of wind and load level. The base case attributes considered are delineated on Table 8 and when the combinations are incorporated, the large number of samples for each totals in excess of $10^{6}$ simulations to ensure adequate sampling of uncertainty distributions. The sequence of hour-ahead to real-time simulation includes the restrictions on ramp limits for operating generators, thereby capturing this important source of emissions attributed to uncertainty. The result of repetitive simulations within the optimization framework are distributions for all system impact metrics, for example production cost, energy price, wind utilization and $\mathrm{CO}_{2}$ emissions, as provided in the results of Section 5.

The two-stage modeling framework is implemented on a case study using the IEEE 39-bus test system, shown in Figure 1. The figure shows the location and distribution of various generation technologies, as described in detail in section 2

The analysis considers the impact of each of the BSER strategies across a number of system scenarios. The scenarios includes the base case system with no wind generation, followed by increases to $10 \%$ and $20 \%$ wind penetration. Other parameters include wind and load forecast levels, and transmission constraints in the network, as well as recourse to demand response. The detailed combination of these scenarios is outlined in Table 8 . Specifics for the transmission constraint modeling are presented in Section 4.6 .

\section{Results and Discussion}

In analyzing the system behavior with building blocks 2, 3 and 4 from EPA's proposed system for emission reduction, the performance measure of prime interest is the resulting reduction in $\mathrm{CO}_{2}$ emissions, while being aware of other potential impacts such as increased production cost and decreased reliability. The results below discuss each of these impacts: $\mathrm{CO}_{2}$ emissions, cost and reliability. The benefit achieved through implementation of real-time demand response is 
also discussed, along with an inefficient solution for emission reduction obtained via transmission constraints.

\subsection{Available Wind Power}

Figure 3 shows the distributions of wind power available to the system at the $10 \%$ and $20 \%$ penetration levels, starting with the forecasted wind levels from the hour ahead market stage, adjusted by the real-time forecast errors. The distributions for wind generation are used in a Monte Carlo framework, see Section 3, to illustrate the potential outcomes over all combinations of forecast level for wind and load, BSER building blocks, and other integration strategies. These combinations, in conjunction with a large number of simulations for each scenario, results in total simulations on the order of $10^{6}$. While computationally expensive, this large number of simulations is required to observe realistic patterns of system response.

\subsection{Emission Reduction through Wind Power}

With the main motivating factor for increasing the use of wind power being its role in decreasing system emissions, the first question for the system analysis is to measure the extent of emission reductions from installing wind power. Figures 4,5 and 6 show the test system $\mathrm{CO}_{2}$ emissions with no wind, $10 \%$ wind and $20 \%$ wind penetration. All scenarios include load forecast errors and generator outages, which account for the variability in emission levels for medium and high load in Figure 4. At low load levels, there is sufficient excess capacity to allow the system to maintain a constant emission level. At medium and high load levels, the power system faces limited options in generators available to dispatch, and the next most economically attractive sources are higher emitters. At highest load levels, more expensive generation is required, which also has a lower marginal emission rate per unit generation. The result of these economic drivers is only a small emission increase between medium and high load scenarios.

Comparing Figures 5 and 6 shows the distributions at low, medium and high load levels of decreasing emissions with increasing wind penetration, as expected. The average reductions across load levels, along with the percentage reduction are shown in Table 9. The average across all load levels for $10 \%$ wind is a $20 \%$ reduction in emissions, and for $20 \%$ wind is a $33 \%$ reduction. Thus, the levels of wind penetration modeled contribute significantly toward achieving the target reductions in the Clean Power Plan, though it is unlikely that wind will reach this level of penetration by 2030 .

Facilitating further emission reduction from changing the generating unit merit order (using natural gas-fired plants before coal-fired plants) and from increasing demand-side energy efficiency are examined next, in order to fully investigate EPA's BSER, and also to explore options for $\mathrm{CO}_{2}$ emission reduction beyond only utilizing wind power. 


\subsection{Emission Reduction with a Carbon Price}

Along with the potential for improving fossil fuel-fired unit performance (not modeled here), the EPA's proposed best system addresses methods to reduce the use of higher $\mathrm{CO}_{2}$ emitting units. A variety of methods would be appropriate for simulating the substitution of natural gas-fired plants for coal-fired plants. For the work presented here a carbon price has been employed, as discussed in Section 2.1.

The fossil fuel-fired units included in the analysis, as appropriate for the New England region, are coal, natural gas combined cycle, combustion turbine (natural gas-fired), and oil. The bottom row of Table 10 (red numerals) shows that with the carbon price included, the merit order of the coal and NGCC units is such that the NGCC units will be dispatched before coal-fired units. This switch in merit order will only be effective to the extent that the system has sufficient capacity to let some units remain idle while others serve load.

Figure 7 shows the carbon emissions with and without employing the carbon price, for increasing wind penetration and increasing load. The x-axis shows the three load levels of low, medium and high load (modeled as different percent reserve margin relative to the installed generating capacity). The y-axis shows the $\mathrm{CO}_{2}$ emissions in tons, and lines represent the three different wind penetrations, $0 \%, 10 \%$ and $20 \%$, with the dashed lines showing the change in expected carbon emissions once the carbon price is employed.

For the test system as modeled, the installed generating capacity relative to the regional load is such that all units are needed to serve load (with no wind installed). For low load the dashed blue line (upper line) is just visible below the solid blue line, indicating that the use of the carbon price has marginal impact on switching the merit order and so reducing carbon emissions, at low load. For medium and high load levels the system does not have enough excess generating capacity to see an impact from the use of the carbon price - there is not enough excess capacity for a switch in merit order to be effective. Note that the system generating capacity is purposefully set to just be able to serve load without any wind in the system in order to not have an excessive amount of generating capacity once the wind power is included. For an actual power system, generating units would be retired, to be replaced in some cases by wind farms, and also regional demand would grow. The analysis presented here is based on short-term operations meaning that fossil fuel and nuclear power plants are not retired or constructed, and there is no regional load growth in order to facilitate comparisons across all the system attributes that are changed (see Table 8).

Returning to Figure 7, the lines for $10 \%$ and $20 \%$ wind penetration levels show considerable benefit in terms of $\mathrm{CO}_{2}$ reductions at low load levels, with less benefit as the load increases. These results show that with sufficient generating capacity (i.e., at low load levels) the system does substitute NGCC units for coal-fired units in the dispatch order and so reduces system-wide $\mathrm{CO}_{2}$ emissions. For $10 \%$ wind this substitution is possible only at low load levels. Conversely, at the $20 \%$ wind penetration level there is enough conventional generating capacity 
to allow the use of the $\mathrm{CO}_{2}$ price to decrease emissions for the full range of system load levels. These results highlight the fact that a system must have sufficient capacity margin to allow any change in merit order, as intended by BSER building block 2, to actually lead to a decreased use of coal units.

\subsection{Demand-Side Efficiency and Demand Response}

The EPA proposes the use of demand-side energy efficiency as part of its BSER since energy efficiency programs reduce the overall use of fossil fuel-fired generating units. This section examines the use of energy efficiency measures along with real-time demand response, with the difference being that energy efficiency programs are intended to permanently reduce demand while demand response in a real-time market could simply shift the demand from a peak period to a lower demand period. The analysis presented here does not allow optimization of all hours in sequence, therefore any load shifting from demand response programs is not represented. However this analysis considers the large number of combinations of forecast levels, demand, and recourse variables for the distributions of uncertainty in wind forecast, load forecast and generator outages. These combinations are sufficient to demonstrate synergies in utilizing EPA's building blocks 2, 3 and 4 and suggest that greater emission reduction will be possible with targeted use of real-time demand response programs. In particular, the emission reduction shown when all these elements are used together is greater than the sum of the emission reduction from the individual elements.

The potential impact of the proposed BSER building blocks at $10 \%$ and $20 \%$ wind penetration is illustrated in Figure 8 . Note that these results do not consider the impact of demand response programs as they are not explicitly included in the BSER guidelines. Figure 8 shows that the use of carbon price is effective in emission reduction at low load for both levels of wind penetration, though medium and higher load scenarios carbon taxes alone are not sufficient to cause emission reduction at lower wind penetration (10\%). The lack of effectiveness of carbon prices alone is due to the inability of the system to meet higher demand without fossil-based generation, at lower wind penetration.

An interesting result of this analysis is that the effectiveness of BSER measures may be improved through the use of responsive demand. Figures 9 and 10 show the system utilization of demand response, or lack thereof, for $20 \%$ wind penetration and high load with and without the use of the carbon price. These figures also show the carbon emissions in the bottom panels. Without the carbon price, the system is shown to not use demand response even when it is available (as the $\$ 100 / \mathrm{MWh}$ price is not economic at this point). The change in $\mathrm{CO}_{2}$ emissions for the blue (left-hand) bars in the bottom of Figure 9 show the effect of demand-side energy efficiency. The lack of use of available real-time demand response is because the generating units have low enough marginal cost that they are dispatched before needing to turn to demand response, with the cost assumptions used in this analysis. Figure 10 shows that once the carbon price is employed it becomes economically efficient to also use the available demand response. 
Emission reduction results from employing the carbon price, energy efficiency and demand response together to achieve greater reductions than any measure alone. For example, with $20 \%$ wind power and no carbon price, the system does not utilize available real-time demand response, with the result that DR alone leads to $0 \%$ emission reductions (with the scenarios modeled in this analysis). With the carbon price employed alone, an average of $3 \%$ reduction in emissions is achieved. Used together, the carbon price and demand response achieve an average of $5 \%$ reduction in emissions, which is clearly greater than the sum of the individual reductions. Similar patterns hold for $10 \%$ wind penetration.

\subsection{Impacts on Reliability and Cost}

Whether from employing a carbon price or other means, a reduction in the use of relatively low cost coal-fired units will lead to an increase in production cost and electricity price. An increase in the use of stochastic generating sources such as solar and wind power will typically lead to increased variability in electricity price as well as increased reliability concerns (Cardell and Anderson (2014)). For the analysis presented here, a threat to system reliability, which occurs when the system cannot maintain the supply-demand energy balance, is represented via forced load curtailment at an assumed value of lost load of $\$ 10,000 /$ MWh (Mount et al. (2011); Willis and Garrod (1997); Murillo-Sanchez et al. (2013)).

As described in Section 3 , the analysis presented here uses Monte Carlo Simulation to represent the uncertainty introduced into the system by the generator outages as well as forecast errors in wind and load. In re-balancing supply and demand after these uncertainties have been realized the OPF simulation turns to load curtailment. With the carbon price employed the frequency of load curtailment increases (reliability decreases) marginally, as shown in Table 11 . However, with real-time demand response available as an additional recourse variable to manage variability, the need to resort to load curtailment is eliminated - reliability is restored.

Table 11 shows the effect on both system reliability (via load curtailment) and total production cost, of employing a carbon price and demand response in the system with $10 \%$ and $20 \%$ wind penetration. These results, aggregating the trends seen in the more than $10^{6}$ simulations, highlight the role of the generator ramping constraints that are imposed between first and second stages of the optimization. With natural gas-fired units dispatched before coal-fired units, the natural gas units are operated closer to their maximum output levels. This leaves less capacity available to ramp up and down in response to wind and load variability, and also forces the system to turn more often to coal plants for the ramping response, and to load curtailment. In this way, employing the $\mathrm{CO}_{2}$ price is seen to make ramping capacity less available, with the result that incidents of load curtailment increase with the $\mathrm{CO}_{2}$ price (in the absence of a possible future ramping product).

A potential degradation of system reliability is represented in terms of the frequency of load curtailment and the subsequent price spikes to $\$ 10,000$. This Table also shows that over simulation the large number of simulations, the 
approximately $0.19 \%$ probability of load curtailment with $10 \%$ wind, and the $3.9 \%$ probability with $20 \%$ wind, are eliminated when demand response is made available. The bottom two rows of Table 11 show general trends in terms of increases or decreases in system production cost. With the $\mathrm{CO}_{2}$ cost employed, the cost of emissions is included in the cost of generation and as a result, total production cost increases. With recourse to real time demand response, the increase in production cost is mitigated for wind penetration levels as shown.

To further analyze the the impact of demand response on frequency of load curtailment, a mixture of normals is used to represent bimodal characteristics of the data. To illustrate, Figure 11 shows a histogram that can be characterized as a mixture of normals (shown in the bottom panel) (Titterington et al. (1985); McLachlan and Krishnan (1997)).

Focusing on the right-hand distribution in the bottom of Figure 11, a decrease in the mean of that distribution to well below $\$ 10,000 / \mathrm{MWh}$ indicates the elimination of load curtailment and so an elimination of the threat to reliability. Any further reduction simply indicates an overall decrease in the average electricity price. Table 12 , summarizing the trends from the more than $10^{6}$ simulation results, shows the mean of this right-hand $(\mathrm{RH})$ distribution for the situation with $20 \%$ wind penetration with and without the use of a carbon price, and comparing the mitigating effect of demand response.

For the test system results shown in Table 12 demand response is shown to be effective in that the average electricity price is lower with demand response, with even the higher distribution mean well below the cost of load shedding.

In addition to a concern over reliability impacts, the EPA's Clean Power Plan addresses the need to achieve the target emission reduction at a reasonable cost. The test system used here does not represent an actual power system, and therefore actual production cost values are not reported. However the relative increase and decrease is meaningful. With the fuel and technology costs used in this analysis (Anderson and Cardell (2013)), the $\$ 52 /$ ton carbon price, with no wind installed, imposes a cost increase around 25\%. As shown in Table 11 these increases are mitigated by the availability of demand response, which significantly increases the flexibility of the system to respond to the increased variability from wind power.

\subsection{A Word of Caution: Transmission Constraints}

The performance of the test system was analyzed with transmission lines into buses 21 and 23 constrained, as well as the lines into buses 5, 9 and 11. This configuration is loosely patterned on historical constraints into the Boston metro and southwest Connecticut regions of New England (ISOne (2007, 2013)). The constraints are implemented by setting the maximum allowed power flow (as determined by thermal limits), to be consistent with the constraints identified above. These constraints are present for all load levels and wind generation levels, yet will only be binding, as in an actual system, when the flow required to serve the load pocket would exceed the defined flow limits. With the network constrained in this manner, the Monte Carlo simulations frequently lead to the case in which the relevant load centers do not have ready access to the modeled 
coal-fired generating units. The result of this situation is that a number of the NGCC units are dispatched before the coal units thus resulting in substitution of coal-fired generation with that from NGCC units, as directed by the BSER building block 2 .

Figure 12 shows the $\mathrm{CO}_{2}$ reductions achieved with the change in generating unit merit order caused by transmission constraints rather than by a policy designed to reduce emissions. Though the reduction in emissions itself is beneficial, the transmission constraints impose an inefficient cost on the system. In addition, when the transmission constraints are not binding or have been relieved through new construction, the apparent emission reduction would evaporate.

Therefore, it is important to note that neglecting the role of transmission constraints could produce short-lived emission reduction. This analysis of system performance with transmission constraints shows that emission reductions can be achieved via such inefficient system operation. This would be a concern in terms of distorting apparent system-wide reductions if monitoring were to be performed during times of binding constraints, only to have these constraints relieved at a later time allowing the system to return to a state with higher emissions.

\section{Conclusions and Policy Implications}

This analysis has examined the use of EPA's best system of emission reduction for regulating greenhouse gas emissions from existing power plants, as proposed in the Clean Power Plan. The first building block in the proposed BSER consists of heat rate improvements at the existing power plants, while the remaining three building blocks employ strategies for emission reduction that are outside the fence of the targeted power plants. The analysis in this paper focuses on these final three building blocks, using a carbon price as a proxy for achieving building block 2, installing wind power to address building block 3 and modeling energy efficiency improvements via demand reductions for building block 4 .

Our analysis points to five key policy implications in implementing the BSER (i) increasing penetration of wind power leads to increasing reductions in $\mathrm{CO}_{2}$ emissions (as expected), (ii) BSER building blocks 2 and 4, which result in substitution of NGCC units for coal, and increasing energy efficiency, lead to additional emission reduction, (iii) to maximize efficacy, a carbon price (either defined directly or achieved via market forces) must be high enough both to induce a switch in merit order between NGCC and coal-fired units, and to promote the use of demand response, (iv) implementation of the BSER building blocks together leads to net emission reduction greater than summing the reductions from implemented the options singly, emphasizing the fact that the system for emission reduction works best when implemented as a system, and (v) there is the potential that implementation of the BSER alone, without additional strategies, could threaten system reliability and/or be achieved only with harmful cost and price increases. 
To address these reliability and cost concerns, our analysis includes recourse to the additional options of real-time demand response. The implication of the results from these simulations is that including such a strategy in addition to the BSER could be necessary in order to significantly mitigate the cost and reliability concerns raised by implementation of building blocks 2,3 and 4 .

In conclusion, EPA's proposed BSER is effective in reducing $\mathrm{CO}_{2}$ emissions. Even without heat rate improvements as specified in building block 1, the remaining three building blocks in the BSER will achieve significant reductions. The modeling results presented here demonstrate that the implementation of the BSER as a system leads to aggregate reductions that are greater than the sum of individual emission reductions if the building blocks were to be implemented singly. This result provides motivation for policy makers and regulators to work with stakeholders, who might otherwise focus on one building block alone, to coordinate their efforts in implementing the BSER in its entirety, and so achieve the maximum possible emission reduction. No single entity or stakeholder would be expected to implement all the building blocks of the BSER. Policy incentives to coordinate efforts will lead to the greatest emission reduction.

The results also point out that though effective in reducing emissions, the BSER alone, particularly with building block 3 implemented via significant wind power penetration, could lead to reliability and cost concerns. The results shown here demonstrate that these issues are successfully mitigated with the addition of recourse to real-time demand response. This expanded system for emission reduction leads to even greater reductions in $\mathrm{CO}_{2}$ emissions, significantly decreases price volatility, eliminates concerns for system reliability and also decreases the increase in system production cost brought about through internalizing the emissions costs.

\section{Acknowledgements}

This work was supported by the National Science Foundation, grant number IIA-1243482 (the WINDINSPIRE project), and the U.S. Department of Energy in cooperation with the Consortium for Electric Reliability Technology Solutions (CERTS). 


\section{References}

AB32, 2006. Assembly Bill 32: Global Warming Solutions Act. URL http://www . arb.ca.gov/cc/ab32/ab32.htm

Anderson, C. L., Cardell, J. B., 2013. Wind power uncertainty and power system performance. Engineering 5 (10A). URL DOI : 10.4236/eng. 2013.510A007

Ballantine, J., Cooke, D., Sep 2014. Demand Resources ISO New England Markets, Introduction to Wholesale Electricity Markets (WEM 101). Tech. rep., , URL: http://www.iso-ne.com/support/training/courses/wem101/15demand resources.pdf.

Barbose, G., Dec. 2012. Renewables Portfolio Standards in the United States: A Status Update. 2012 National Summit on RPS, URL: $\quad$ http://www.cesa.org/assets/2012-Files/RPS/RPSSummitDec2012Barbose.pdf.

Bills, G. W., Oct 1970. On-line stability analysis study. EPRI Report RP90-1 for the Edison Electric Institute.

Black, J., Aug. 2013. Outlook for Renewable Resources in New England. ISO New England, Rhode Island Technical Session, 1-29.

CACJA, 2010. Colorado Clean Air, Clean Jobs Act. URL http://www.coga.org/pdfs_facts/CleanAirCleanJobs.pdf

Cardell, J., Anderson, C. L., Jan. 2010. Analysis of the System Costs of Wind Variability Through Monte Carlo Simulation. Proceedings of the 43rd Hawaii International Conference on System Sciences, 1-8.

Cardell, J. B., Anderson, C. L., 2014. A flexible dispatch margin for wind integration. IEEE Transactions on Power Systems, in press.

DSIRE, 2014. Database of State Incentives for Renewables and Efficiency.

URL http://www.dsireusa.org/incentives/index.cfm?state=us

DSIRE Solar Incentives, 2014. Database of State Incentives for Renewables and Efficiency.

URL http://www.dsireusa.org/solar/index. $c f m ? e e=1 \& R E=1 \&$ spf=1\&st= 1

Eilperin, J., Mufson, S., 2014. EPA is readying climate rule for existing power plants as deadline approaches, Washington Post.

URL http://www.washingtonpost.com/politics/ epa-is-readying-climate-rule-for-existing-power-plants-as-... deadline-approaches/2014/05/21/8d1c0b5c-e088-11e3-9743-bb9b59cde7b9_ story.html 
FERC, 2000-2014. Federal Energy Regulatory Commission Form No. 714 - Annual Electric Balancing Authority Area and Planning Area Report. Tech. rep., , URL: http://www.ferc.gov/docs-filing/forms/form-714/data.asp.

FERC, 2012. Ferc staff 2010 iso/rto metrics report, appendix f iso new england (iso-ne). Tech. rep., , URL:http://www.ferc.gov/industries/electric/indusact/rto/metrics/iso-ne-rto-metrics.pdf.

FERC, Aug 2014. Federal Energy Regulatory Commission Staff Report Common Metrics, Docket No. AD14-15-000. Tech. rep.

Hamal, C. W., Sharma, A., Sep. 2011. Adopting a ramp charge to improve performance of the ontario market. LECG, 1-53.

Heeter, J., Barbose, G., Bird, L., Weaver, S., Flores-Espino, F., Kuskova-Burns, K., Wiser, R., 2014. A Survey of State-Level Cost and Benefit Estimates of Renewable Portfolio Standards. Lawrence Berkeley Nationl Laboratory; National Renewable Energy Laboratory LBNL-6589e.

ISOne, 2006. 2006 demand response programs summary table, iso new england, inc. Tech. rep.

ISOne, Jun 2007. 2006 annual markets report, iso new england inc. Tech. rep., , URL: http://www.isone.com/markets/mktanlysrpts/annlmktrpts/2006/2006annualmarketsreport.pdf.

ISOne, Nov 2013. 2013 regional system plan, iso new england inc. Tech. rep., uRL: http://iso-ne.com/staticassets/documents/trans/rsp/2013/rsp13_final.docx.

Klobasa, M., 2010. Analysis of demand response and wind integration in Germany's electricity market. IET Renewable Power Generation 4 (1).

Lalor, G., O’Malley, M., Jun 2003. Frequency Control on an Island Power System with Increasing Proportions of CCGT. 2003 IEEE Power Tech Conference Proceedings 4.

Lange, M., 2005. On the Uncertainty of Wind Power Predictions-Analysis of the Forecast Accuracy and Statistical Distribution of Errors. Journal of Solar Energy Engineering 127 (2), 177.

Lindsay, J., Dragoon, K., Aug. 2010. Summary Report on Coal Plant Dynamic Performance Capability. Tech. rep., Renewable Northwest Project.

Luckow, P., Stanton, E., Biewald, B., Fisher, J., Ackerman, F., Hausman, E., Feb. 2014. 2013 Carbon Dioxide Price Forecast. Synapse Energy Economic, Inc., 1-27.

McLachlan, G., Krishnan, T., 1997. The EM Algorithm and Extensions. Wiley. 
Mount, T., Lamadrid, A., Maneevitjit, S., Jul 2011. The economic value of transmission lines with increased penetrations of stochastic generation. 2011 IEEE Power and Energy Society General Meeting, 1-8.

Murillo-Sanchez, C., Zimmerman, R., Lindsay Anderson, C., Thomas, R., Dec 2013. Secure Planning and Operations of Systems With Stochastic Sources, Energy Storage, and Active Demand. IEEE Transactions on Smart Grid 4 (4), 2220-2229.

NACAA, Jul. 2011. Background and History of EPA Regulation of Greenhouse Gas (GHG) Emissions Under the Clean Air Act \& National Association of Clean Air Agencies' Comments on EPA GHG Regulatory and Policy Proposals. National Association of Clean Air Agencies, 1-12.

NERC, March 2013. Nerc 2011 demand response availability report. Tech. rep., , URL: http://www.nerc.com/docs/pc/dadswg/2011 DADS Report.pdf.

NPCC, Aug. 2002. Natural Gas Combined Cycle Gas Turbine Performance. NPPC Working Group, 1-16.

NREL, 2010. Wind systems integration eastern wind integration and transmission study. Tech. rep., National Renewable Energy Laboratory.

Perlstein, B., Battenberg, L., Gilbert, E., Maslowski, R., Stern, F., Schare, S., Corfee, K., Firestone, R., 2012. Potential Role of Demand Response Resources in Maintaining Grid Stability and Integrating Variable Renewable Energy under California's 33 Percent Renewable Portfolio Standard. California's Demand Response Measurement and Evaluation Committee.

RDI/FT Energy Inc., 2006. PowerDat database. RDI/FT Energy Inc.

RGGI, 2012. Regional Greenhouse Gas Initiative, Program Review. URL http://www.rggi.org/

Shahidehpour, Wang, Mar. 2004. Optimal generation scheduling with ramping costs . IEEE Power Industry Computer Application Conference, 1-7.

Shrestha, G. B., Song, K., Goel, L., Aug. 2004. Strategic Self-Dispatch Considering Ramping Costs in Deregulated Power Markets. IEEE Transactions on Power Systems 19 (3), 1575-1581.

Tee, C. Y., Cardell, J., Ellis, G., Oct 2009. Short-term load forecasting using artificial neural networks. In: North American Power Symposium (NAPS), 2009. pp. 1-6.

Titterington, D. M., Smith, A. F. M., Makov, U. E., 1985. Statistical Analysis of Finite Mixture Distributions. Wiley. 
UCS, 2014. Union of Concerned Scientists, Production Tax Credit for Renewable Energy.

URL http://www.ucsusa.org/clean_energy/smart-energy-solutions/ increase-renewables/production-tax-credit-for.html

US DOE/EIA, Jun. 2012. US Department of Energy, Energy Information Administration, Annual Energy Outlook, DOE/EIA-0383.

URL http://www.eia.gov/forecasts/aeo/pdf/0383\%282012\%29.pdf

US EPA, Jun. 2014a. US Environmental Protection Agency, Carbon Pollution Emission Guidelines for Existing Stationary Sources: Electric Utility Generating Units. Federal Register 40 CFR Part 60, 1-645, URL: https://www.federalregister.gov/articles/2014/06/18/2014-13726/carbonpollution-emission-guidelines-for-existing-stationary-sources-electric-utilitygenerating.

US EPA, 2014b. US Environmental Protection Agency, Clean Power Plan Proposed State Goals.

URL http://www2.epa.gov/sites/production/files/2014-06/ 20140602-state-data-summary.xlsx

US EPA, 2014c. US Environmental Protection Agency, GHG Abatement Measures Technical Support Document.

URL http://www2.epa.gov/sites/production/files/2014-06/ documents/20140602tsd-ghg-abatement-measures.pdf

US EPA, Jun. 2014d. US Environmental Protection Agency, Goal Computation Technical Support Document. EPA Technical Support Document, 1-29.

US EPA, Jan. 2014e. US Environmental Protection Agency, Standards of Performance for Greenhouse Gas Emissions From New Stationary Sources: Electric Utility Generating Units. Federal Register, 40 CFR Parts 60, 70, 71, and 98 79 (5), 1-91, URL: http://yosemite.epa.gov/opei/rulegate.nsf/byrin/2060aq91.

US EPA, May 2014f. US Environmental Protection Agency, State Plan Considerations Technical Support Document, 1-136.

URL http://www2.epa.gov/sites/production/files/2014-06/ documents/20140602tsd-state-plan-considerations.pdf

US EPA, OAR, May 2011. US Environmental Protection Agency, Inventory of U.S. Greenhouse Gas Emissions and Sinks, Fast Facts, 1-2.

US Supreme Court, 2005. Massachusetts, et al., Petitioners v. Environmental Protection Agency, et al. 549 U.S. 497127 S. Ct. 1438, 167 L. Ed. 2d 248.

Willis, K. G., Garrod, G. D., 1997. Electricity supply reliability: Estimating the value of lost load. Energy Policy 25 (1), 97103.

Zimmerman, R. D., Murillo-Sanchez, C. E., Gan, D., May 2010. Matpower 4.0b4 User's Manual. Power Systems Engineering Research Center. 


\section{List of Figures}

$1 \quad$ IEEE 39-bus Test System One-Line Diagram with Technology Mix 23

$2 \quad$ Simulation Flow Diagram $\ldots \ldots \ldots \ldots \ldots \ldots$

$3 \quad$ Wind Generation Histograms . . . . . . . . . . . . . . . 25

$4 \quad \mathrm{CO}_{2}$ Emissions with No Wind Installed $\ldots \ldots \ldots \ldots$

$5 \quad \mathrm{CO}_{2}$ Emissions with $10 \%$ Wind $\ldots \ldots \ldots \ldots . \ldots . \ldots 27$

$6 \quad \mathrm{CO}_{2}$ Emissions with $20 \%$ Wind $\ldots \ldots \ldots \ldots . \ldots . \ldots . \ldots$

$7 \quad \mathrm{CO}_{2}$ Emissions with $\mathrm{CO}_{2}$ Price $\ldots \ldots \ldots \ldots \ldots \ldots$

$8 \quad$ Impact of BSER on $\mathrm{CO}_{2}$ emissions at $10 \%$ (top) \& $20 \%$ (bottom) wind penetration $\ldots \ldots \ldots \ldots \ldots \ldots \ldots \ldots$

9 Use of DR and $\mathrm{CO}_{2}$ Reduction without Carbon Price . . . . . . 31

10 Use of DR and $\mathrm{CO}_{2}$ Reduction with Carbon Price . . . . . . . . 32

$11 \quad$ Mixture of Normals Example . . . . . . . . . . . . . . 33

$\begin{array}{lll}12 & \mathrm{CO}_{2} \text { Emission Reduction with Transmission Constraints } \ldots . . .34\end{array}$ 
Figure 1: IEEE 39-bus Test System One-Line Diagram with Technology Mix

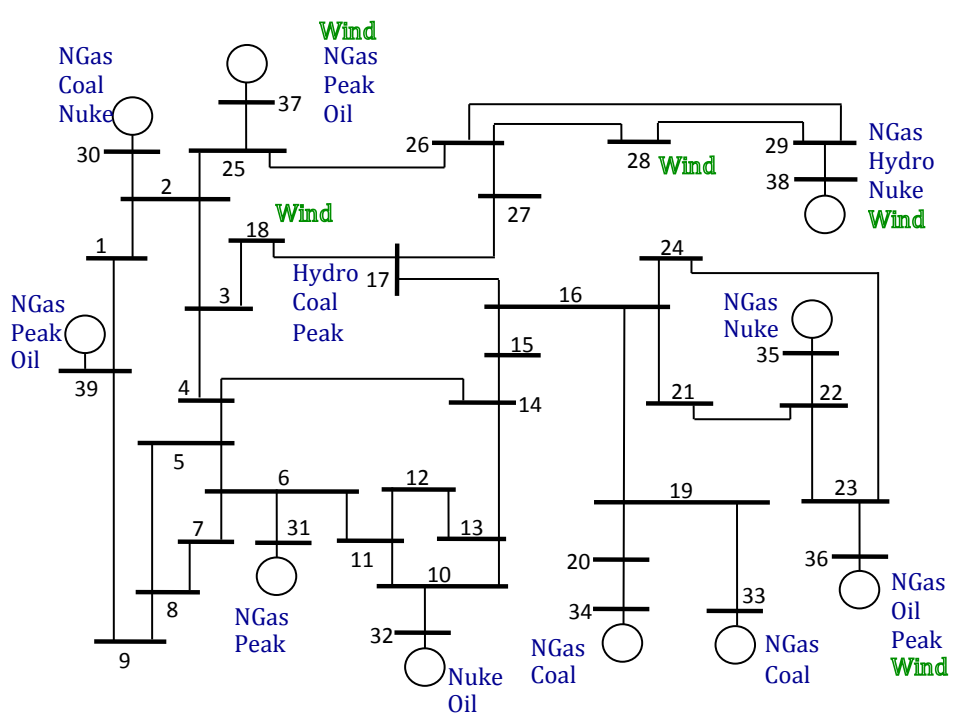


Figure 2: Simulation Flow Diagram

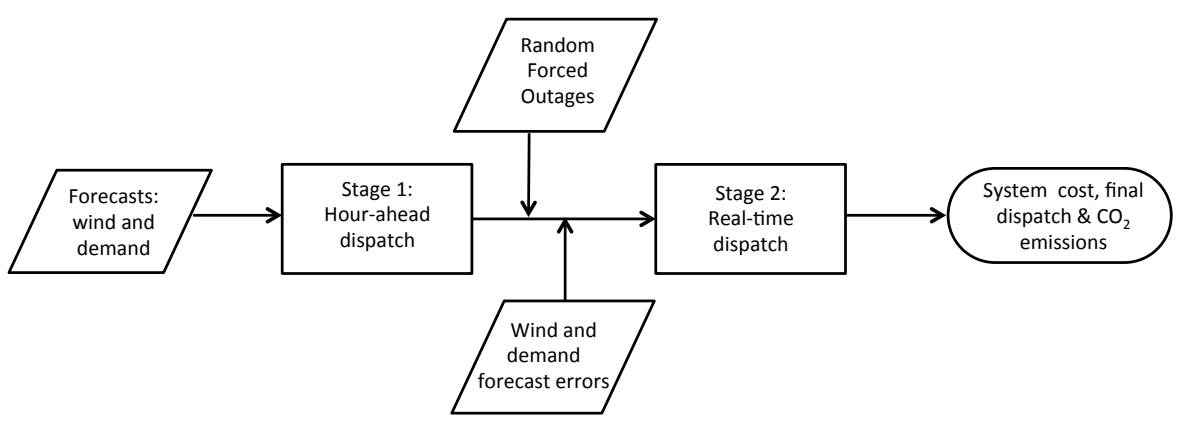


Figure 3: Wind Generation Histograms

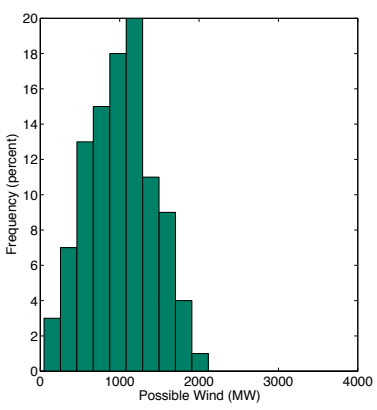

(a) $10 \%$ Wind Penetration

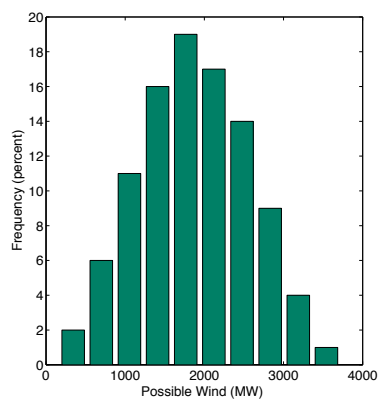

(b) $20 \%$ Wind Penetration 
Figure 4: $\mathrm{CO}_{2}$ Emissions with No Wind Installed

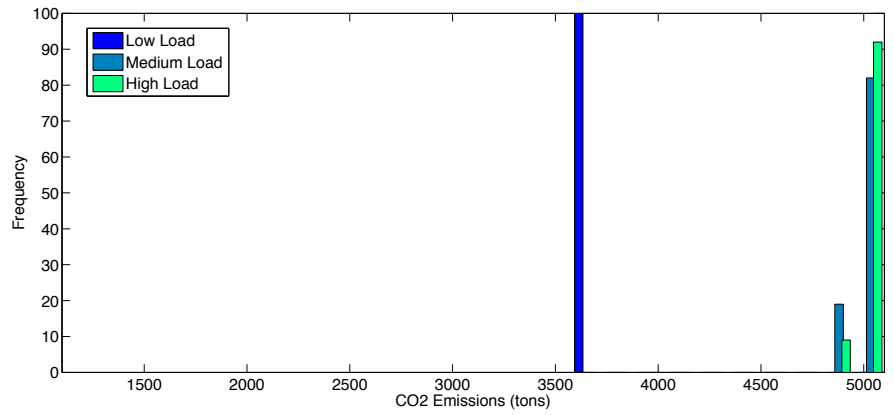


Figure 5: $\mathrm{CO}_{2}$ Emissions with 10\% Wind

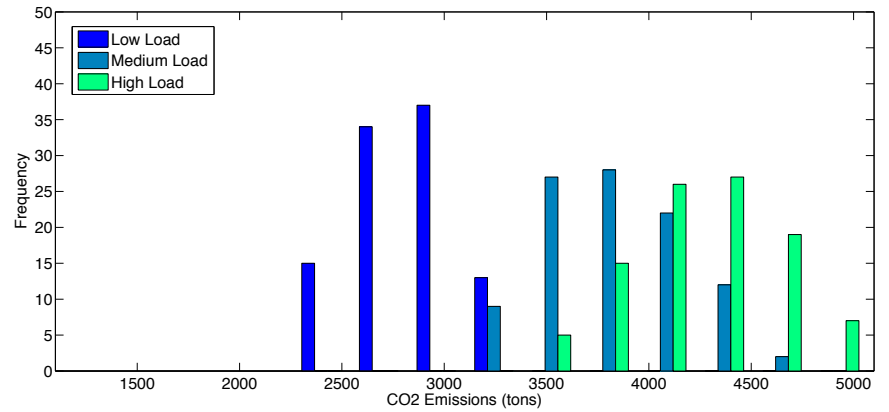


Figure 6: $\mathrm{CO}_{2}$ Emissions with 20\% Wind

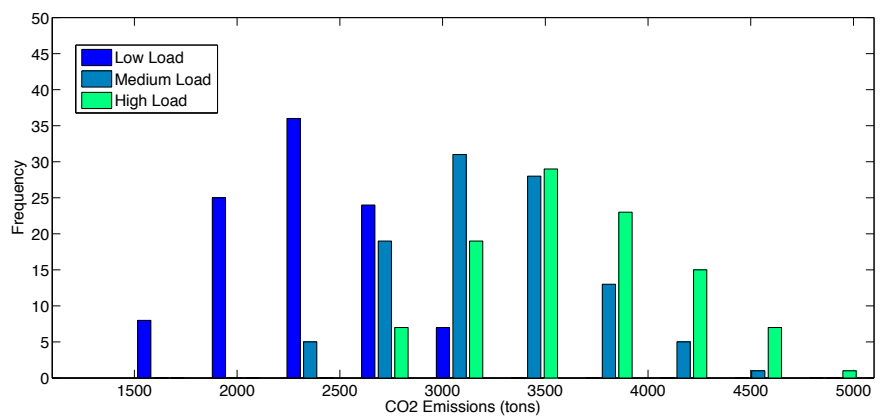


Figure 7: $\mathrm{CO}_{2}$ Emissions with $\mathrm{CO}_{2}$ Price

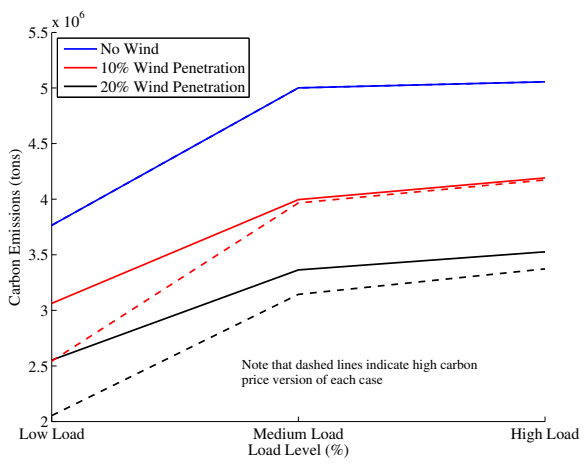


Figure 8: Impact of BSER on $\mathrm{CO}_{2}$ emissions at $10 \%$ (top) \& $20 \%$ (bottom) wind penetration
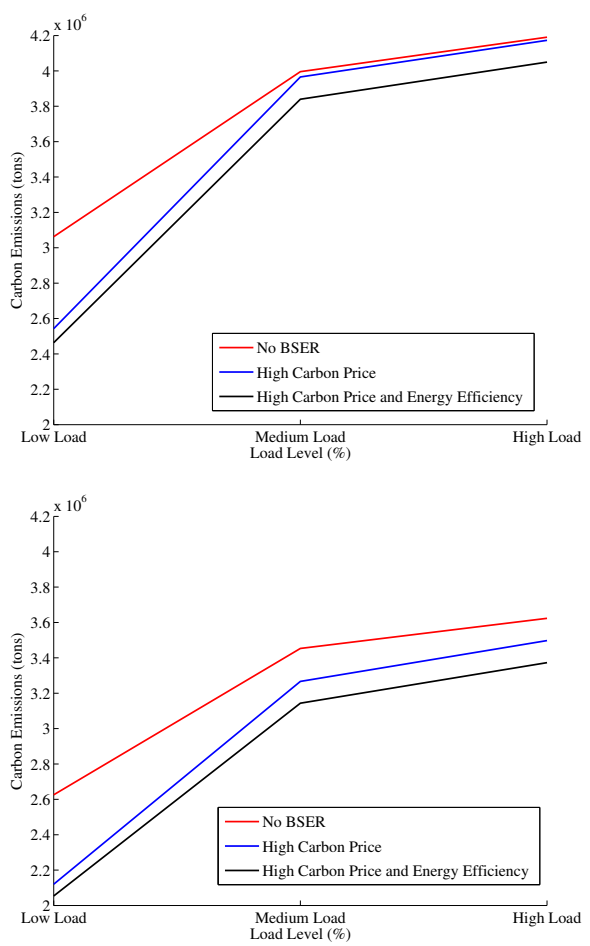
Figure 9: Use of DR and $\mathrm{CO}_{2}$ Reduction without Carbon Price
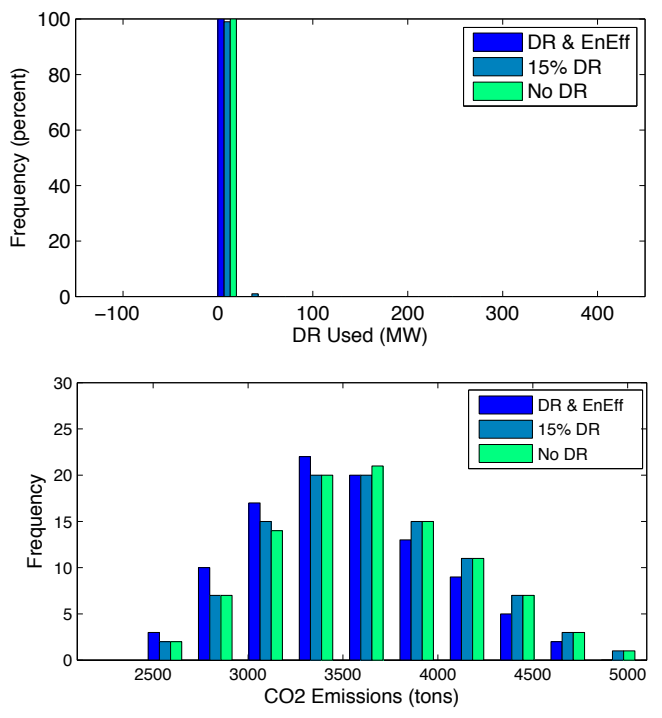
Figure 10: Use of DR and $\mathrm{CO}_{2}$ Reduction with Carbon Price
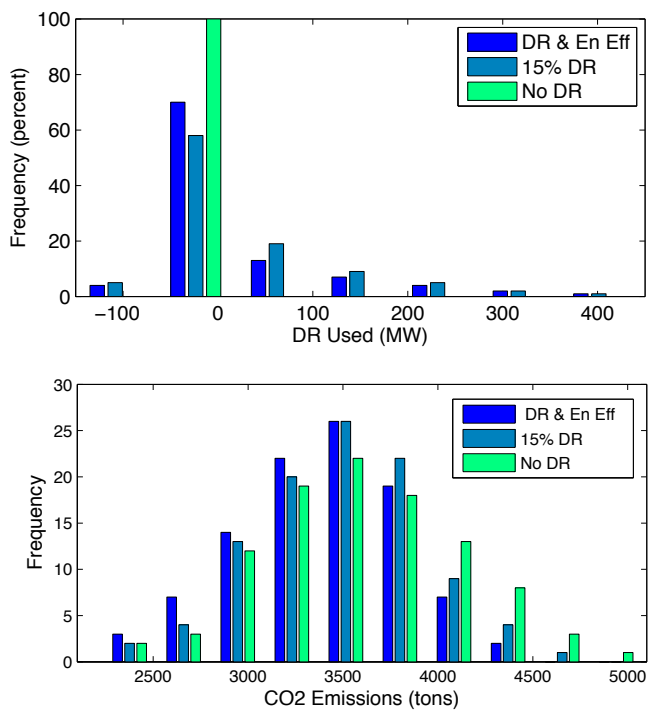
Figure 11: Mixture of Normals Example
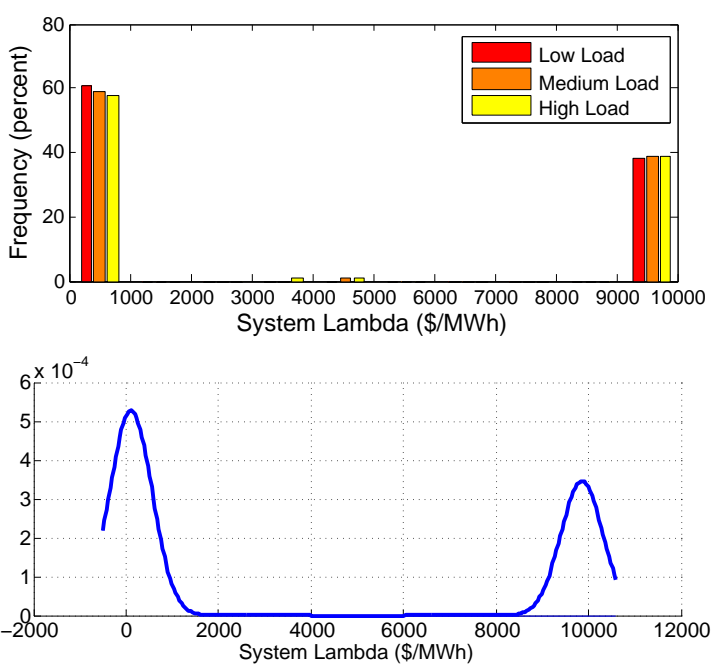
Figure 12: $\mathrm{CO}_{2}$ Emission Reduction with Transmission Constraints

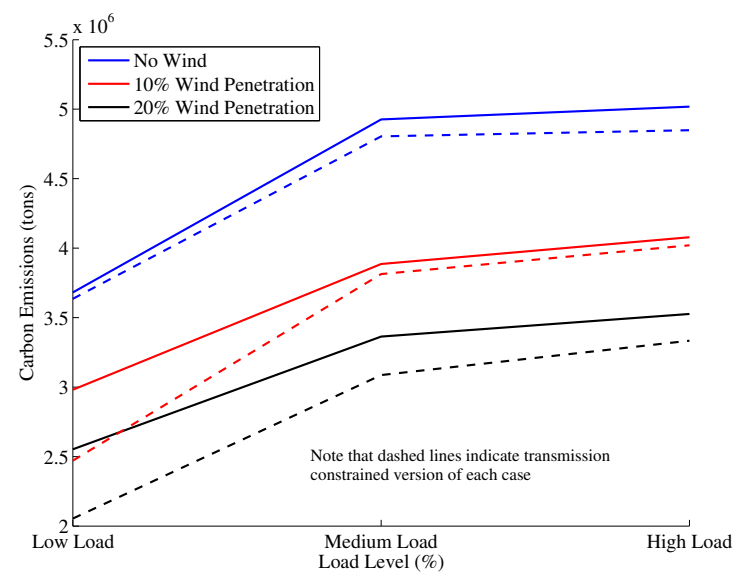




\section{List of Tables}

$1 \quad$ Target $\mathrm{CO}_{2}$ Reductions Under CAA $\S 111(\mathrm{~d}) \ldots \ldots \ldots \ldots$

$2 \quad$ Target Renewable Energy Generation, BSER Building Block 3 . 37

$3 \quad$ Target Demand-Side Energy Efficiency, BSER Building Block 4 . 38

$4 \quad$ Generator Technology Mix for 39 Bus Test System, (MW)

$5 \quad$ Generator Costs, Carbon Content and Applied Carbon Price . . 40

$6 \quad$ Modeled Installed Wind (GW) (assuming 30\% capacity factor) . 41

$7 \quad$ Generation Technology Ramping Capability $\ldots \ldots \ldots$. . . . . . . . 42

$8 \quad$ Modeling Scenarios . . . . . . . . . . . . . . . . . . . . 43

$9 \quad \mathrm{CO}_{2}$ Reductions with Wind (lbs) $\ldots \ldots \ldots \ldots \ldots \ldots$

$10 \quad$ Generating Unit Variable Costs with \& without Carbon Price . . 45

11 Cost and Reliability with Wind, Carbon Price \& Demand Response 46

12 Mean System $\lambda$ for RH Distribution: Role of DR . . . . . . . 47 
Table 1: Target $\mathrm{CO}_{2}$ Reductions Under CAA $\S 111(\mathrm{~d})$

\begin{tabular}{lcc}
\hline STATE & FinAl GoAL $^{a}$ & $\left(\mathrm{LBS} \mathrm{CO}_{2} / \mathrm{MWH}\right)^{b}$ \\
\hline Connecticut & $29 \%$ & 540 \\
Maine & $14 \%$ & 378 \\
Massachusetts & $38 \%$ & 576 \\
New Hampshire & $46 \%$ & 486 \\
Rhode Island & $14 \%$ & 782 \\
Vermont ${ }^{c}$ & $*$ & $*$ \\
\hline North Dakota $(\min \%)$ & $11 \%$ & 1,783 \\
Washington $(\max \%)$ & $72 \%$ & 215 \\
\hline
\end{tabular}

${ }^{a}$ Reductions to be achieved by 2030 (interim goals beginning in 2020) (US EPA (2014a))

${ }^{\circ}$ Adjusted output-weighted-avg $\mathrm{lbs} \mathrm{CO}_{2} /$ net MWh from all affected fossil fuel-fired generating units (US EPA $(2014 \mathrm{f} / \mathrm{b})$ )

${ }^{c}$ The asterisk indicates: "Because Vermont and the District of Columbia lack affected sources, no goals are being proposed for these jurisdictions." US EPA (2014a) p 39. 
Table 2: Target Renewable Energy Generation, BSER Building Block 3

\begin{tabular}{lrr}
\hline StATE & 2012 & FinAl GOAL \\
\hline Connecticut & $2 \%$ & $9 \%$ \\
Maine & $28 \%$ & $25 \%$ \\
Massachusetts & $5 \%$ & $24 \%$ \\
New Hampshire & $7 \%$ & $25 \%$ \\
Rhode Island & $1 \%$ & $6 \%$ \\
Vermont & $*$ & $*$ \\
\hline North Dakota & $15 \%$ & $15 \%$ \\
Washington & $7 \%$ & $15 \%$ \\
\hline
\end{tabular}


Table 3: Target Demand-Side Energy Efficiency, BSER Building Block 4

\begin{tabular}{lrr}
\hline StATE & 2012 & FINAL GOAI \\
\hline Connecticut & $13.4 \%$ & $11.9 \%$ \\
Maine & $5.4 \%$ & $12.1 \%$ \\
Massachusetts & $6.3 \%$ & $11.8 \%$ \\
New Hampshire & $4.9 \%$ & $11.0 \%$ \\
Rhode Island & $11.2 \%$ & $11.6 \%$ \\
Vermont & $15.4 \%$ & $*$ \\
\hline North Dakota & $1.4 \%$ & $9.7 \%$ \\
Washington & $4.2 \%$ & $11.3 \%$ \\
\hline
\end{tabular}

${ }^{a}$ Cumulative savings as a percent of retail sales. 
Table 4: Generator Technology Mix for 39 Bus Test System, (MW)

\begin{tabular}{lrrrrrr}
\hline & COAL & OIL & CT & NGCC & NUCLEAR & HYDRO \\
\hline North & 80 & 205 & 125 & 890 & 390 & 135 \\
Mass & 245 & 600 & 215 & 1600 & 575 & 250 \\
South & 90 & 410 & 280 & 910 & 370 & 0 \\
\hline
\end{tabular}


Table 5: Generator Costs, Carbon Content and Applied Carbon Price

\begin{tabular}{lrrrr}
\hline \$/MWH & COAL & OIL & NGCC & CT \\
\hline Carbon Content $(\mathrm{kg} / \mathrm{MWh})$ & 1004 & 824 & 538 & 902 \\
Fixed Cost & 69.2 & 24.6 & 19.4 & 49.5 \\
Variable Cost & 24.3 & 115.0 & 45.6 & 71.5 \\
Var + Carbon Price & 81.8 & 162.2 & 76.4 & 123.2 \\
\hline
\end{tabular}


Table 6: Modeled Installed Wind (GW) (assuming 30\% capacity factor)

\begin{tabular}{lrrrrr}
\hline WIND \% & Bus 37 & Bus 18 & Bus 38 & Bus 28 & Total (GW) \\
\hline $10 \%$ & 0.80 & 0.85 & 0.5 & 0.0 & 2.15 \\
$20 \%$ & 1.00 & 1.00 & 0.5 & 1.5 & 4.00 \\
\hline
\end{tabular}


Table 7: Generation Technology Ramping Capability

\begin{tabular}{lcc}
\hline TECHNOLOGY & $\begin{array}{c}\text { Minimum } \\
(\% / \text { minute })\end{array}$ & $\begin{array}{c}\text { MaXimum } \\
(\% / \text { minute })\end{array}$ \\
\hline Coal & 0.6 to 1.2 & 2.4 to 2.7 \\
Oil & 0.5 & 4.0 \\
NGas & 0.8 & 3.0 \\
Peak & 7.0 & 30.0 \\
Hydro & 30.0 & 50.0 to 100.0 \\
Nuclear & not used & not used \\
\hline
\end{tabular}


Table 8: Modeling Scenarios

\begin{tabular}{llll}
\hline ATTRIBUTE & OPTIONS & & \\
\hline Wind Penetration & None & $10 \%$ & $20 \%$ \\
Wind Forecast & High $(>85 \%)$ & Medium & Low $(<11 \%)$ \\
Load Level & High & Medium & Low \\
DSM & Nothing & Efficiency & DR+Efficiency \\
Tx Constraints & Yes & No & \\
\hline
\end{tabular}


Table 9: $\mathrm{CO}_{2}$ Reductions with Wind (lbs)

\begin{tabular}{lrrr}
\hline & LOW LOAD & MED LOAD & HiGH LOAD \\
\hline No Wind & 3650 & 5005 & 5020 \\
\hline 10\% Wind & 2815 & 3825 & 4260 \\
\% Reduction & $-23 \%$ & $-24 \%$ & $-15 \%$ \\
\hline $20 \%$ Wind & 2345 & 3240 & 3610 \\
\% Reduction & $-36 \%$ & $-35 \%$ & $-28 \%$ \\
\hline
\end{tabular}


Table 10: Generating Unit Variable Costs with \& without Carbon Price

\begin{tabular}{lrrrr}
\hline \$/MWH & COAL & NGCC & NGCT & OIL \\
\hline Variable Cost & 24.3 & 45.6 & 71.5 & 115.0 \\
Var $+\mathrm{CO}_{2}$ Price & 81.8 & 76.4 & 123.2 & 162.2 \\
\hline
\end{tabular}


Table 11: Cost and Reliability with Wind, Carbon Price \& Demand Response

\begin{tabular}{lccc}
\hline & Base Case & $\mathrm{CO}_{2}$ Price & $\&$ with DR \\
\hline Load Curtail, 10\% wind & $0.18 \%$ & $0.19 \%$ & eliminated \\
Load Curtail, 20\% wind & $3.6 \%$ & $3.9 \%$ & eliminated \\
\hline Prod. Cost, 10\% wind & - & $\Uparrow$ & $\downarrow 20 \%$ of increase \\
Prod. Cost, 20\% wind & - & $\Uparrow$ & $\downarrow 11 \%$ of increase \\
\hline
\end{tabular}


Table 12: Mean System $\lambda$ for RH Distribution: Role of DR

\begin{tabular}{lrr}
\hline & $\mathrm{No} \mathrm{CO}_{2}$ Price & $\mathrm{CO}_{2}$ Price \\
\hline $20 \%$ Wind Base Case & $\$ 10,000$ & $\$ 10,000$ \\
with DR & $\$ 60$ & $\$ 90$ \\
\hline
\end{tabular}

